Open Access to Pharmaceutical and Medical Research

(C) 2011-20, publisher and licensee JDDT, This is an Open Access article which permits unrestricted non-commercial use, provided the original work is properly cited

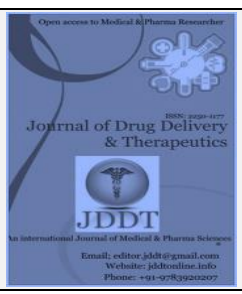

Open Access

Research Article

\title{
Pharmacist-Led Interventions on Improving Outcomes in Patients with Diabetes Mellitus: Evidence from the Literature
}

\author{
Sini T Inasu1,2* and MV. Kumudavalli1 \\ ${ }^{1}$ Vinayaka Missions College of Pharmacy, Vinayaka Mission's Research Foundation (Deemed University) Salem, Tamil Nadu- 636008, India \\ 2 Department of Pharmacy Practice, Ahalia School of Pharmacy, Palakkad, Kerala- 678557, India
}

\begin{abstract}
Diabetes mellitus is a rapidly growing major health problem world-wide. The management of type 2 diabetes mellitus is complex, requiring continuous medical care by health care professionals and considerable self-care efforts by patients. A collaborative and integrated team approach in which pharmacists can play a pivotal role should be sought when managing patients with diabetes. Pharmacist-led care programs have been shown to help patients with diabetes succeed in achieving treatment goals and improving outcomes. Hence, the aim of this narrative review is to address and summarize the effectiveness of pharmacist interventions in the management of diabetic patients. A comprehensive literature search was conducted in PubMed/Medline, Scopus, web of Science and the Cochrane Library were searched from the date of database inception to June 2019. All randomized controlled trials evaluating the effectiveness of pharmacist-based interventions on diabetic patients in comparison with usual care were included in study. Outcomes of interest included short-term and long-term measures such as glycated haemoglobin (HbA1c), and secondary outcomes were blood glucose level, blood pressure (BP), lipid profile, body mass index (BM I), 10-year coronary heart disease (CHD) risk, medication adherence, health related quality of life (HRQoL), and economic outcomes. Twenty-five studies were included in this systematic study. They were heterogeneous in terms of interventions, participants, settings and outcomes. Pharmacist-led self-management interventions included education on diabetes and its complications, medication adherence, lifestyle and education of selfmanagement skills. Few studies even focussed on patients need through a tailored intervention. We found that those who received the pharmacist care had a statistically significant improvement in HbA1C, blood pressure, lipid profile, health-related quality of life, and CHD risk. These results underline the added value of pharmacists in patient-related care. Hence this review supports the involvement of pharmacists as a member of health- care teams in managing diabetic patients at diverse settings worldwide.
\end{abstract}

Keywords: diabetes, self-management, HbA1C, pharmaceutical care, randomized controlled trial

Article Info: Received 26 April 2020; Review Completed 17 June 2020; Accepted 25 June 2020; Available online 15 July 2020

Cite this article as:

Inasu ST, Kumudavalli MV, Pharmacist-Led Interventions on Improving Outcomes in Patients with Diabetes Mellitus: Evidence from the Literature, Journal of Drug Delivery and Therapeutics. 2020; 10(4):49-58 http://dx.doi.org/10.22270/jddt.v10i4.4195

*Address for Correspondence:

Sini T Inasu, Assistant professor, Department of Pharmacy Practice, Ahalia School of Pharmacy, Palakkad, Kerala- 678557, India

\section{INTRODUCTION}

Diabetes mellitus is a major chronic health problem for decades to come. Indeed, it was estimated that 463 million people globally suffered from diabetes in 2019 , and this number is predicted to increase to 700 million people by 2045.1 Diabetes, if left uncontrolled, may cause microvascular and macrovascular complications in the long term, which are the main causes of increased morbidity and mortality and decreased health-related quality of life among patients. ${ }^{2}$

The management of diabetic mellitus is very difficult and it became a persisted task all over the world. ${ }^{3}$ Overtime, several studies have shown that prolonged high levels of glcaemia lead to micro- and macrovascular complications. ${ }^{4}$ The overall goal of diabetes treatment is, therefore, to reduce the risk of diabetes-related complications by achieving and maintaining near normal glycaemic control with lifestyle changes and drug therapy ${ }^{5}$. Despite the benefits of effective therapies, literature demonstrates that achievement of desired therapeutic outcomes in patients with diabetes remains suboptimal that could increase the disease burden.6,7 Barriers to adherence may consists of complex treatment regimens, medication side effects, poor patient-provider communication, socio-economic issues, memory impairment psychological well-being and personal beliefs. ${ }^{8}$

Due to the complexities associated with managing diabetes population, new models of health care delivery should be developed and implemented for better glycaemic control.9,10 Thus, evidences has shown that a multidisciplinary team 
with collaboration among different healthcare professionals can provide a more holistic treatment and to obtain better outcomes. ${ }^{11}$ A larger role of the pharmacist, in diabetes treatment by helping patients improve their chances of reaching therapeutic and lifestyle goals. The responsibilities of pharmacist involve patient education, monitoring treatment goals, adherence, drug-related problem assessment.12 They can positively influence patients individually or with other health professionals in improving diabetes management by providing pharmaceutical care programs and prudent pharmacological therapy to improve disease state outcomes. ${ }^{13}$ Several studies have recommended the involvement of a pharmacist in a diabetes multidisciplinary healthcare team including those by the American Diabetes Association and Canadian Diabetes Association. ${ }^{12,14}$ Previous literature reviews have proven that the contribution of pharmacists in achieving better control of diabetes is significant ${ }^{15-16}$. These reviews focused on many types of pharmacist interventions including selfcare related interventions, adherence, and compliance or on counselling but always resulted in a significant effect on the outcomes of diabetes mellitus ${ }^{17-18}$.

In this study, a narrative and systematic review was performed to determine the relative efficacy of various pharmacist based interventions involving diabetes education alone and in combination with pharmaceutical care, and those interventions in which diabetes education was provided by health care team including pharmacist as team member, on clinical outcomes of the type 2 diabetes patients. We choose to use glycosylated hemoglobin (HbA1c) as primary outcome, as it has been shown to be a good surrogate marker for diabetes related complications ${ }^{19}$. Other secondary outcomes include fasting blood sugar (FBS), body mass index (BMI), blood pressure control (BP), cardiovascular incidences (CV) and lipid profile.

\section{MATERIALS AND METHODS}

\section{Study design}

A comprehensive literature search was done in PubMed/Medline, Scopus, web of Science and the Cochrane Library from the date of database inception to November 2019. The PubMed search strategy served as a reference for the development of search strategies for the remaining database. The standardized search strategy included the use of medical subject headings terms or text words related to pharmacist interventions (pharmacists, pharmaceutical care, medication therapy management, pharmaceutical services); to the disease (diabetes, diabetes mellitus type2, glycosylated haemoglobin, glycaemia, blood glucose, glycaemic); and to clinical trials (randomized controlled trial, controlled clinical trial, random allocation). The electronic database searches were complemented by manually reviewing the reference of relevant reviews and included studies.

\section{Inclusion criteria}

Studies were included in this review if they were randomized controlled trials or cluster-randomized controlled trials evaluating the effectiveness of interventions delivered only or mainly by pharmacists and directed at patients with type 2 diabetes in comparison with usual care. Studies that took place in a community pharmacy and in outpatient primary care and hospital settings were also included. Studies were included if they reported one or more outcome measures were reported, for example: glycosylated haemoglobin (A1c); blood glucose (fasting, postprandial, or random); blood pressure; lipid profile (total cholesterol, low-density lipoprotein cholesterol [LDL], high-density lipoprotein cholesterol [HDL], and triglycerides; body mass index (BMI), medication adherence or health-related quality of life (HRQoL). It must be an original study published in a peer-reviewed journal and the full text article was published in English language.

\section{Study selection}

Two independent reviewers screened all titles and abstracts retrieved from the electronic databases using the predefined inclusion criteria. Then, the full text of each potentially eligible article was obtained and screened independently by two reviewers to determine their eligibility for further analysis. Any discrepancies regarding study inclusion was resolved through discussion.

\section{Data extraction and Synthesis}

One of the researchers extracted the data from the studies included in this review. No blinding for author or journal was applied in the extraction process. The data extracted from the studies includes publication details (title, authors, publication year and journal name); study design characteristics (country where the study took place, type of the study, sample size, period of study); study characteristics (patients age, gender, follow-up duration, details of pharmacist interventions and usual care, inclusion and exclusion criteria) and study outcomes. The description of the intervention includes counselling on diabetes education, quality of life, medication details, lifestyle modifications, patient compliance toward the treatment, and clinical outcomes (HbA1c, fasting and random blood glucose levels, $\mathrm{BP}, \mathrm{BMI}$, and lipid profile). Pharmacist interventions were evaluated in all the included studies. Outcomes in the form of HbA1c, random and fasting glucose levels, BP, BMI, and lipid profiles were evaluated in all included studies. The risk of bias in the included studies was measured by two independent reviewers according to pre-defined criteria based on the Cochrane risk-of-bias tool (Rob 2.0). Any divergences were deliberated till consensus was reached. Results for all the outcomes (HbA1c, FBS, BMI, CV incidence, $\mathrm{BP}$, lipid profile etc) and intervention across the included studies were analysed and described narratively.

\section{RESULTS}

In total 5919 articles were identified from the electronic database searches. On the basis of title and abstract evaluation 3930 studies were excluded as they did not meet the inclusion criteria of the study. The full text of 66 papers was assessed, with 25 papers finally being included in the review. (Figure 1). The main characteristics and study population of the included studies are presented in Table 1.

\section{Study characteristics}

The studies included were heterogenous in terms of interventions, participants, settings and outcomes. They varied in their quality and reporting of their findings conducted in several setting including: standard care, pharmaceutical care or family practice in the UK, Spain or elsewhere in the world20-28. Among the included studies 24 were randomised controlled studies ${ }^{20-35,37-44}$ and one was cluster randomised ${ }^{36}$. All studies were published from 2009 onwards. Most of the included studies $(n=12)$ were conducted in Asia,22 2427283034353940414344 followed by Europe ( $\mathrm{n}=5$ ),, 131333638 North America ( $=$ 5),,25 26293242 South America $(\mathrm{n}=2), 2337$ and Africa $(\mathrm{n}=1) \cdot 30$

The median follow-up time was 6 months, $22,23,25,28,30,33,36,37,38,40,41,42,44$ two studies had a followup time of less than 6 months 27,39 and ten of more than 6 months. ${ }^{20,21,24,26,29,31,32,34,35,43}$ 
The majority of the studies focused primarily on diabetes mellitus type 2 patients, ${ }^{20,22-28,30,31,33-44}(\mathrm{n}=22)$, one study included both type 1 and type 2 patients, ${ }^{32}$ and one study did not specify the type of diabetes. ${ }^{29}$

\section{Description of Intervention}

The settings in which the studies took place included community pharmacies, primary care clinics or health centres, and hospitals. The nature of interventions provided by pharmacist varied among the included studies and covered one or more of the following topics: counselling and education on diabetes, medication, lifestyle modification, and self-monitoring; reinforcement of medication adherence or complications screening; provision of materials such as educational leaflets and pill boxes; medication review; identification and resolution of drug-related problems; discussions with the primary care provider regarding pharmacotherapy; adjustment of pharmacotherapy; and referrals to other health care professionals. Two studies mentioned motivational interviews as a technique used to deliver advice to patients. ${ }^{20,28}$ In most studies, the control group received usual care from medical and nursing staff and/or community pharmacists, depending on the study setting.

The intervention in the included studies were all provided by a trained pharmacist, either by pharmacist alone 20-24,26-
$33,35-37,39,40,43,44$ or within a multi-disciplinary team. ${ }^{25,34,41,42}$ one study did not specify the intervention team, besides including a pharmacist. ${ }^{38}$ Most interventions targeted the individual patient20-24,26,28-33,35-40,43,44 whereas some interventions used group sessions $25,34,41,42$. One study did not specify whether the intervention was offered in an individual or group setting27.

Thirteen studies included diabetes education as interventions ${ }^{21-23,25,28,30,31,36,39-42,44}$ either about diabetes in general or about acute and chronic complications. Patient education on medication was provided by the pharmacists in 16 included studies and this included education about adherence, dosage, drug-related problems, indication, storage, and use $20-29,31,37-40,42,44$.

In eighteen studies the intervention included selfmanagement skills support20-22,25-27,29-32,34,38-44 and in around 10 studies participants were trained in self-monitoring glucose. $22,23,25,28,30-32,39,40,42$

In most of the studies, education was given on lifestyle modifications, exercise requirements, foot cares, management of proper diet and smoking cessation were included as the part of the intervention by the pharmacists. Other interventions such as the use of a diabetes diary and provision of written educational material were reported in the included studies.

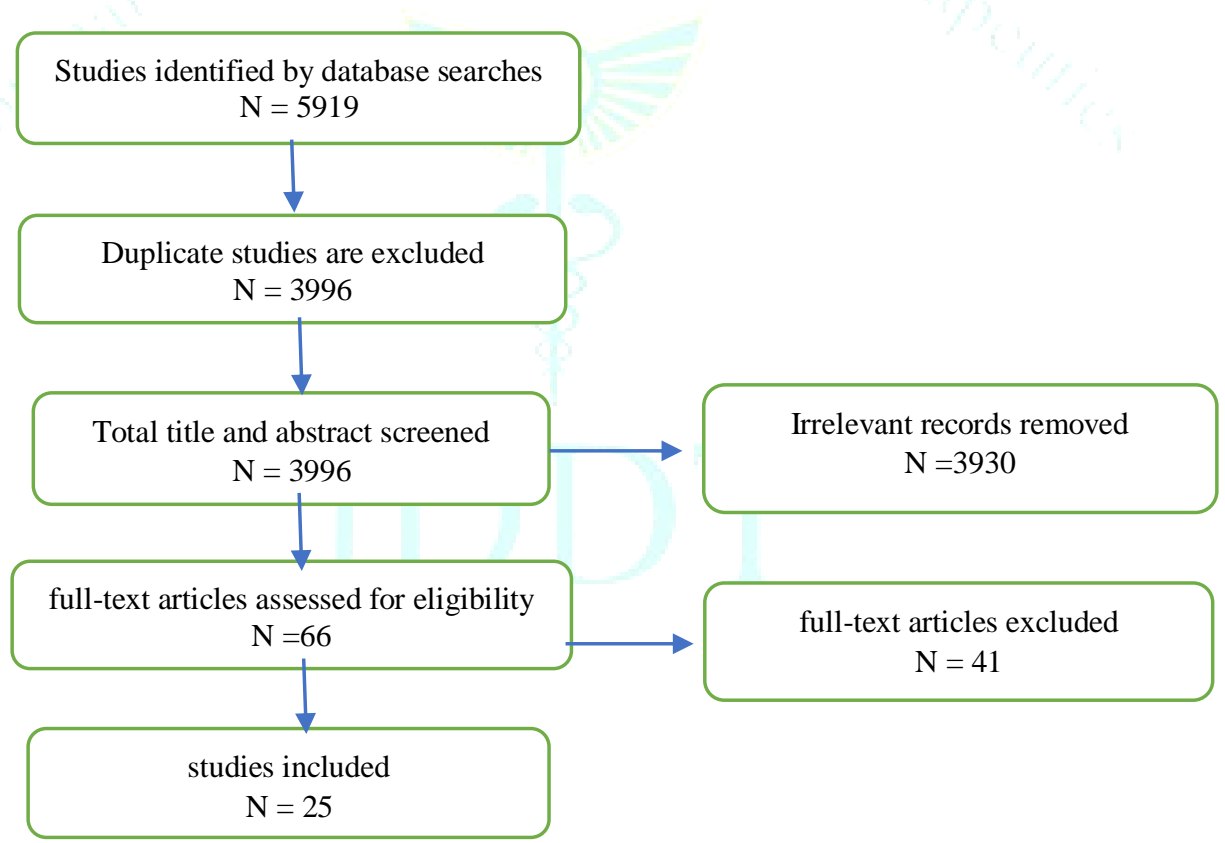

Figure 1: Flow chart of study selection

\section{DISCUSSION}

This review found evidence of the importance of the clinical pharmacist/ pharmacist care program that targeted immediate and long-term risk factors associated with diabetes mellitus. This extended across multiple health care settings and cultures worldwide. In most of the included studies, pharmacist's role was mainly to specify all drug related problem including poor drug compliance and side effects and communicating these to the physician. such rapport is crucial in building professional working relations necessary to ensure optimal patient care. However, pharmacist involvement is not meant to replace the formal diabetes education or physician direct care, nevertheless, the program provides a usual supplement or enhancement to the care of diabetic patients.

The type, intensity, and frequency of the interventions were different in all the included studies. The number of visits for face-to-face interactions varied once in a week to once in a year. Some of the included studies do have face-to face contact with the pharmacists or pharmacy departments $20,22,35$ and some have combination of face toface contacts along with telephone contact with the pharmacists or department of pharmacy. ${ }^{25,28}$ The evidence indicate that telephone interventions are effective and significant improvement in glycaemic level.45 
Table No.1: Characteristics of the included studies.

\begin{tabular}{|c|c|c|c|c|c|c|}
\hline $\begin{array}{l}\text { Author, Year, } \\
\text { Country }\end{array}$ & Study design & $\begin{array}{l}\text { No. of Patients } \\
\text { Lost to Follow-up } \\
\text { Age in Years (Mean } \\
\text { [SD]) } \\
\text { Gender (\%) }\end{array}$ & $\begin{array}{l}\text { Follow- } \\
\text { up } \\
\text { Duration }\end{array}$ & $\begin{array}{l}\text { Pharmacist } \\
\text { Intervention }\end{array}$ & Control & $\begin{array}{l}\text { Outcome } \\
\text { measure }\end{array}$ \\
\hline $\begin{array}{l}\text { Adibe et al. } \\
2013^{20} \\
\text { Nigeria }\end{array}$ & $\begin{array}{l}\text { Randomized, } \\
\text { controlled } \\
\text { study }\end{array}$ & $\begin{array}{l}\text { No. of patients } \\
\text { (IG/CG):110/110 } \\
\text { Lost to follow-up } \\
\text { (IG/CG):11/17 } \\
\text { Age (IG/CG): } \\
52.4[7.6] / 52.8 \text { [8.2] } \\
\text { Gender (IG/CG): } \\
44.6 \% / 40.0 \% \text { male }\end{array}$ & 12 months & $\begin{array}{l}\text { Pharmaceutical care } \\
\text { including education on } \\
\text { diabetes, self-monitoring, } \\
\text { medication, lifestyle } \\
\text { modification, counselling, } \\
\text { and effective interaction } \\
\text { with health providers }\end{array}$ & $\begin{array}{l}\text { Usual care } \\
\text { offered by } \\
\text { hospitals }\end{array}$ & $\begin{array}{l}\text { HbA1c. LDL, } \\
\text { CVD risks, } \\
\text { HRQoL, } \\
\text { medication } \\
\text { understanding, } \\
\text { cost-utility } \\
\text { analysis }\end{array}$ \\
\hline $\begin{array}{l}\text { Ali et al. } \\
2012 \\
\text { UK } 21\end{array}$ & $\begin{array}{l}\text { Randomized, } \\
\text { controlled } \\
\text { study }\end{array}$ & $\begin{array}{l}\text { No. of patients } \\
\text { (IG/CG): } 25 / 23 \\
\text { Lost to follow-up } \\
\text { (IG/CG): } 2 / 0 \\
\text { Age (IG/CG): } \\
66.4[12.7] / 66.8[10.2] \\
\text { Gender }(\mathrm{IG} / \mathrm{CG}): \\
43.5 \% / 56.5 \% \text { male }\end{array}$ & 12 months & $\begin{array}{l}\text { Pharmaceutical care } \\
\text { including medicine use } \\
\text { review; education } \\
\text { regarding diabetes and its } \\
\text { complications; and } \\
\text { counselling on lifestyle } \\
\text { modification with referral } \\
\text { to other health care } \\
\text { professional when } \\
\text { appropriate }\end{array}$ & $\begin{array}{l}\text { Usual } \\
\text { service } \\
\text { from } \\
\text { general } \\
\text { practition } \\
\text { er, } \\
\text { practice } \\
\text { nurse, and } \\
\text { communit } \\
\text { y } \\
\text { pharmacy }\end{array}$ & $\begin{array}{l}\text { A1c, BG, SBP, } \\
\text { DBP, TC, LDL, } \\
\text { HDL, TG, BMI, } \\
\text { DQoL, HRQoL, } \\
\text { diabetes } \\
\text { knowledge, } \\
\text { SIMS, beliefs } \\
\text { about } \\
\text { medicines, } \\
\text { other }\end{array}$ \\
\hline $\begin{array}{l}\text { Butt et al. } \\
2016^{22} \\
\text { Malaysia }\end{array}$ & $\begin{array}{l}\text { Randomized, } \\
\text { controlled } \\
\text { study }\end{array}$ & $\begin{array}{l}\text { No. of Patients } \\
\text { (IG/CG):33/33 } \\
\text { Lost to follow-up } \\
\text { (IG/CG):4/3 } \\
\text { Age (IG/CG) } 57.42 \\
\text { [7.17]/57.12[10.78] } \\
\text { Gender (IG/CG): } \\
60.6 / 57.6\end{array}$ & 6 months & $\begin{array}{l}\text { Pharmaceutical care } \\
\text { including education on } \\
\text { diabetes and its } \\
\text { complication, self- } \\
\text { management education, } \\
\text { counselling on medication } \\
\text { use and medication } \\
\text { adherence }\end{array}$ & Usual care & $\begin{array}{l}\text { HbA1c, FBS, } \\
\text { BMI, TC, HDL-C, } \\
\text { LDL- C, } \\
\text { triglycerides, } \\
\text { QoL, diabetes } \\
\text { education and } \\
\text { MMMAS scores }\end{array}$ \\
\hline $\begin{array}{l}\text { Cani et al } \\
2015^{23} \\
\text { Brazil }\end{array}$ & $\begin{array}{l}\text { Parallel } \\
\text { randomized } \\
\text { controlled } \\
\text { trial }\end{array}$ & $\begin{array}{l}\text { No. of Patients } \\
\text { (IG/CG):37/41 } \\
\text { Lost to follow-up } \\
\text { (IG/CG):3/5 } \\
\text { Age(IG/CG): } \\
61.91(9.58) / 61.58(8.14) \\
\text { Gender } \\
\text { (IG/CG):61.7/61.1 }\end{array}$ & 6 months & $\begin{array}{l}\text { Individualized } \\
\text { pharmacotherapeutic } \\
\text { care plan consisting of } \\
\text { education about diabetes, } \\
\text { education on lifestyle } \\
\text { modification and } \\
\text { provision of free } \\
\text { glucometer and pill } \\
\text { counter }\end{array}$ & $\begin{array}{l}\text { Standard } \\
\text { care }\end{array}$ & $\begin{array}{l}\text { HbA1c, FBS, } \\
\text { BMI, QoL, } \\
\text { medication } \\
\text { adherence, } \\
\text { insulin injection } \\
\text { and home blood } \\
\text { glucose } \\
\text { monitoring } \\
\text { techniques, } \\
\text { diabetes } \\
\text { education }\end{array}$ \\
\hline $\begin{array}{l}\text { Chan et al. } \\
201224 \\
\text { Hong Kong }\end{array}$ & $\begin{array}{l}\text { Parallel } \\
\text { randomized } \\
\text { controlled } \\
\text { trial }\end{array}$ & $\begin{array}{l}\text { No. of Patients } \\
\text { (IG/CG):51/54 } \\
\text { Lost to follow-up } \\
\text { (IG/CG):0/0 } \\
\text { Age (IG/CG): } \\
63.2(9.5) / 61.7(11.2) \\
\text { Gender (IG/CG) } \\
\text { female(\%): } 41.2 / 48.1\end{array}$ & 9 months & $\begin{array}{l}\text { Pharmaceutical care } \\
\text { program addressing } \\
\text { medication adherence, } \\
\text { knowledge and beliefs, } \\
\text { skills, perceived health } \\
\text { and cognitive functions, } \\
\text { and identification of DRPs }\end{array}$ & $\begin{array}{l}\text { Routine } \\
\text { medical } \\
\text { care }\end{array}$ & $\begin{array}{l}\text { HbA1c, SBP, } \\
\text { DBP, TC, LDL, } \\
\text { HDL, TG, BMI, } \\
\text { Albumin- } \\
\text { creatinine ratio, } \\
\text { CHD risk, stroke } \\
\text { risk medication } \\
\text { knowledge, } \\
\text { medication } \\
\text { adherence, cost- } \\
\text { effectiveness } \\
\text { analysis }\end{array}$ \\
\hline $\begin{array}{l}\text { Cohen et al. } \\
2011^{25} \\
\text { USA }\end{array}$ & $\begin{array}{l}\text { Randomized } \\
\text { controlled } \\
\text { trial }\end{array}$ & $\begin{array}{l}\text { No. of patients } \\
\text { (IG/CG):53/50 } \\
\text { Lost to follow-up } \\
\text { (IG/CG): } 5 / 2 \\
\text { Age (IG/CG): }\end{array}$ & 6 months & $\begin{array}{l}\text { Part of a multidisciplinary } \\
\text { diabetes specific healthy } \\
\text { lifestyle education } \\
\text { intervention and } \\
\text { behavioural and } \\
\text { pharmacologic } \\
\text { interventions, including }\end{array}$ & $\begin{array}{l}\text { Standard } \\
\text { primary } \\
\text { care }\end{array}$ & $\begin{array}{l}\text { HbA1c, SBP, } \\
\text { LDL, HRQoL, } \\
\text { perceived } \\
\text { competence, } \\
\text { adherence to } \\
\text { self-care } \\
\text { activities, }\end{array}$ \\
\hline
\end{tabular}




\begin{tabular}{|c|c|c|c|c|c|c|}
\hline & & $\begin{array}{l}\text { 69.8[10.7]/67.2 [9.4] } \\
\text { Gender (IG/CG): } \\
\text { 100.0/96.0\% male }\end{array}$ & & medication changes & & $\begin{array}{l}\text { disease } \\
\text { knowledge }\end{array}$ \\
\hline $\begin{array}{l}\text { Doucette et } \\
\text { al. } 200926 \\
\text { USA }\end{array}$ & $\begin{array}{l}\text { Parallel } \\
\text { randomised } \\
\text { controlled } \\
\text { trial }\end{array}$ & $\begin{array}{l}\text { No. of Patients } \\
\text { (IG/CG):36/42 } \\
\text { Lost to follow-up } \\
\text { (IG/CG):5/7 } \\
\text { Age (IG/CG): } \\
\text { 58.7(13.3)/61.2(10.9) } \\
\text { Gender (IG/CG): } \\
61.8 / 53.7 \% \text { male }\end{array}$ & 12 months & $\begin{array}{l}\text { Pharmaceutical care } \\
\text { including education on } \\
\text { diabetes, self-monitoring } \\
\text { and pharmacologic } \\
\text { interventions including } \\
\text { medication changes }\end{array}$ & $\begin{array}{l}\text { Standard } \\
\text { primary } \\
\text { care }\end{array}$ & $\begin{array}{l}\mathrm{HbA1c}, \mathrm{BP}, \mathrm{LDL}- \\
\mathrm{C} \text {, adherence to } \\
\text { self-care } \\
\text { activities }\end{array}$ \\
\hline $\begin{array}{l}\text { Farsaei et al } \\
2011^{27} \\
\text { Iran }\end{array}$ & $\begin{array}{l}\text { Parallel } \\
\text { randomized } \\
\text { controlled } \\
\text { study }\end{array}$ & $\begin{array}{l}\text { No. of patients } \\
\text { (IG/CG):87/87 } \\
\text { Lost to follow-up: NR } \\
\text { Age (IG/CG): } \\
\text { 53.4[9.8]/52.9 [8.5] } \\
\text { Gender(IG/CG): } \\
63.2 \% / 68.2 \% \text { female }\end{array}$ & 3 months & $\begin{array}{l}\text { Pharmacist-led program } \\
\text { including education on } \\
\text { medications adherence } \\
\text { and self-management and } \\
\text { provision of SMBG data } \\
\text { entry log book }\end{array}$ & $\begin{array}{l}\text { General } \\
\text { education } \\
\text { offered by } \\
\text { the } \\
\text { nursing } \\
\text { staff }\end{array}$ & HbA1c, FBG \\
\hline $\begin{array}{l}\text { Jarab et al } \\
2012^{28} \\
\text { Jordan }\end{array}$ & $\begin{array}{l}\text { Parallel } \\
\text { randomized } \\
\text { controlled } \\
\text { study }\end{array}$ & $\begin{array}{l}\text { No. of patients } \\
\text { (IG/CG):85/86 } \\
\text { Lost to follow-up } \\
\text { (IG/CG):8/7 } \\
\text { Age (IG/CG): } 63.4 \\
\text { [10.1]/65.3 [9.2] } \\
\text { Gender (IG/CG): } \\
42.4 / 44.2 \% \text { female }\end{array}$ & 6 months & $\begin{array}{l}\text { Comprehensive clinical } \\
\text { pharmacy care including } \\
\text { education on diabetes, } \\
\text { medication and lifestyle } \\
\text { changes; review of } \\
\text { prescribed treatment; } \\
\text { referral to a smoking } \\
\text { cessation program; and } \\
\text { provision of booklet } \\
\text { about diabetes } \\
\text { medications and lifestyle } \\
\text { changes }\end{array}$ & Usual care & $\begin{array}{l}\text { HbA1c, FBS, BP, } \\
\text { lipid levels BMI, } \\
\text { diabetes self- } \\
\text { care activities, } \\
\text { medication } \\
\text { adherence }\end{array}$ \\
\hline $\begin{array}{l}\text { Jameson et } \\
\text { al } \\
2010^{29} \\
\text { USA }\end{array}$ & $\begin{array}{l}\text { Parallel } \\
\text { randomized } \\
\text { controlled } \\
\text { study }\end{array}$ & $\begin{array}{l}\text { No. of Patients } \\
\text { (IG/CG):52/51 } \\
\text { Lost to follow-up (IG/CG): } \\
\text { not mentioned } \\
\text { Age (IG/CG): } \\
\text { 49.3(10.8)/49.7(10.9) } \\
\text { Gender (IG/CG): } \\
\text { ]51.1/5\% female }\end{array}$ & 12 months & $\begin{array}{l}\text { Evaluation and } \\
\text { adjustment of therapeutic } \\
\text { regimen, and patient } \\
\text { education on self -care } \\
\text { activities, medication and } \\
\text { insulin, and medication } \\
\text { adherence }\end{array}$ & Usual care & HbA1c \\
\hline $\begin{array}{l}\text { Jahangard- } \\
\text { Rafsanjani et } \\
\text { al. } \\
201530 \\
\text { Iran }\end{array}$ & $\begin{array}{l}\text { Parallel } \\
\text { randomized } \\
\text { controlled } \\
\text { study }\end{array}$ & $\begin{array}{l}\text { No. of Patients } \\
\text { (IG/CG):51/50 } \\
\text { Lost to follow-up } \\
\text { (IG/CG):6/10 } \\
\text { Age (IG/CG): } \\
57.3(8.6) / 55.9(8.7) \\
\text { Gender (IG/CG): } \\
\text { 49/52\%Female }\end{array}$ & 6 months & $\begin{array}{l}\text { Pharmaceutical care } \\
\text { involving education about } \\
\text { diabetes and its } \\
\text { complication and on self- } \\
\text { management, counselling } \\
\text { on medication adherence, } \\
\text { provision of written } \\
\text { educational material and } \\
\text { SMBG data entry log book }\end{array}$ & Usual care & $\begin{array}{l}\text { HbA1c, BP, } \\
\text { Medication } \\
\text { adherence, } \\
\text { Diabetes self- } \\
\text { care activity, } \\
\text { BMI, } \\
\text { satisfaction, } \\
\text { diabetes } \\
\text { knowledge }\end{array}$ \\
\hline $\begin{array}{l}\text { Korcegez et } \\
\text { al. } \\
2017^{31} \\
\text { Cyprus }\end{array}$ & $\begin{array}{l}\text { Parallel } \\
\text { randomized } \\
\text { controlled } \\
\text { study }\end{array}$ & $\begin{array}{l}\text { No. of Patients } \\
\text { (IG/CG):79/80 } \\
\text { Lost to follow-up } \\
\text { (IG/CG):4/3 } \\
\text { Age(IG/CG): } \\
61.80 \pm 10.38 / 62.22 \pm 9.54 \\
\text { Gender (IG/CG) } \\
\text { female(\%):77.3/74 }\end{array}$ & 12 months & $\begin{array}{l}\text { Pharmaceutical care } \\
\text { including education about } \\
\text { diabetes and its } \\
\text { complication, self- } \\
\text { management and on } \\
\text { lifestyle modification, } \\
\text { counselling for } \\
\text { medication use, provision } \\
\text { of written educational } \\
\text { material }\end{array}$ & Usual care & $\begin{array}{l}\text { HbA1c, FBS, } \\
\text { SBP, DBP, TC, } \\
\text { LDL-C, HDL-C, } \\
\text { TGs, BMI, } \\
\text { Medication } \\
\text { adherences, } \\
\text { diabetes } \\
\text { knowledge, self- } \\
\text { care activities }\end{array}$ \\
\hline $\begin{array}{l}\text { Kraemer et } \\
\text { al. } 2012^{32} \\
\text { USA }\end{array}$ & $\begin{array}{l}\text { Parallel } \\
\text { randomized } \\
\text { controlled } \\
\text { study }\end{array}$ & $\begin{array}{l}\text { No. of Patients } \\
\text { (IG/CG):36/31 } \\
\text { Lost to follow-up }\end{array}$ & 12 months & $\begin{array}{l}\text { pharmacist counselling } \\
\text { and empowering with } \\
\text { diabetes education and its } \\
\text { complication, self- } \\
\text { management and on }\end{array}$ & $\begin{array}{l}\text { Usual care } \\
\text { with } \\
\text { written } \\
\text { education } \\
\text { al }\end{array}$ & $\begin{array}{l}\text { HbA1c, FBS, } \\
\text { SBP, DBP, TC, } \\
\text { LDL-C, HDL-C, } \\
\text { TGs, BMI }\end{array}$ \\
\hline
\end{tabular}




\begin{tabular}{|c|c|c|c|c|c|c|}
\hline & & $\begin{array}{l}\text { (IG/CG):1/1 } \\
\text { Age(IG/CG): } \\
55.6(6.8) / 52.6(9.2) \\
\text { Gender (IG/CG) } \\
\text { female(\%): } 38.8 / 61.29\end{array}$ & & $\begin{array}{l}\text { lifestyle modification, } \\
\text { medication use, provision } \\
\text { of written educational } \\
\text { material }\end{array}$ & $\begin{array}{l}\text { informatio } \\
\mathrm{n} \text { about } \\
\text { managing } \\
\text { diabetes }\end{array}$ & \\
\hline $\begin{array}{l}\text { Kjeldsen et } \\
\text { al. } 201533 \\
\text { Denmark }\end{array}$ & $\begin{array}{l}\text { Randomized } \\
\text { controlled } \\
\text { study }\end{array}$ & $\begin{array}{l}\text { No. of patients } \\
\text { (IG/CG):41/125 } \\
\text { Lost to follow-up } \\
\text { (IG/CG):4/23 } \\
\text { Age (IG/CG): } 63.4 \\
\text { [7.8]/62.1 [10.2] } \\
\text { Gender (IG/CG): } \\
\text { 59.5/62.4\% male }\end{array}$ & 6 months & $\begin{array}{l}\text { Pharmaceutical care by } \\
\text { nonadherence screening, } \\
\text { medication review, } \\
\text { patient education and } \\
\text { coaching regarding } \\
\text { metabolic syndrome, } \\
\text { support to structure and } \\
\text { remember medication } \\
\text { intake, feedback to PCP, } \\
\text { and referral to other } \\
\text { health services }\end{array}$ & NR & $\begin{array}{l}\text { BG, SBP, HRQoL, } \\
\text { disease } \\
\text { knowledge, } \\
\text { medication } \\
\text { adherence, } \\
\text { patient } \\
\text { satisfaction with } \\
\text { service, others }\end{array}$ \\
\hline $\begin{array}{l}\text { Lau et al. } \\
2018^{34} \\
\text { Malaysia }\end{array}$ & $\begin{array}{l}\text { Pilot } \\
\text { retrospective } \\
\text { cohort study }\end{array}$ & $\begin{array}{l}\text { No. of Patients } \\
\text { (IG/CG):29/29 } \\
\text { Lost to follow-up } \\
\text { (IG/CG):0/0 } \\
\text { Age (IG/CG): } 54.24 \\
\text { (9.97)/ 59.07 (10.25) } \\
\text { Gender (IG/CG): } \\
48.1 / 51.9 \% \text { female }\end{array}$ & 12 months & $\begin{array}{l}\text { Multidisciplinary } \\
\text { collaborative care by } \\
\text { educating patients } \\
\text { regarding diabetes } \\
\text { control, nutrition } \\
\text { restriction and } \\
\text { requirement, } \\
\text { management of co- } \\
\text { morbid conditions, and } \\
\text { lifestyle modification. } \\
\text { medication adjustment, }\end{array}$ & $\begin{array}{l}\text { Standard } \\
\text { primary } \\
\text { care }\end{array}$ & $\begin{array}{l}\text { HbA1c, diabetic } \\
\text { related } \\
\text { hospitalization }\end{array}$ \\
\hline $\begin{array}{l}\text { Lim et al. } \\
201635 \\
\text { Malaysia }\end{array}$ & $\begin{array}{l}\text { Prospective } \\
\text { randomized } \\
\text { open-labelled } \\
\text { study }\end{array}$ & $\begin{array}{l}\text { No. of Patients } \\
\text { (IG/CG):50/50 } \\
\text { Lost to follow-up } \\
\text { (IG/CG):11/13 } \\
\text { Age (IG/CG): } \\
55.62(1.49) / 57.00(1.56) \\
\text { Gender(IG/CG): } \\
53.8 / 54.1 \% \text { female }\end{array}$ & 12 months & $\begin{array}{l}\text { Education regarding life } \\
\text { style modification, } \\
\text { diabetes and its } \\
\text { complication, counselling } \\
\text { for medication use and } \\
\text { medication adherence } \\
\text { and provision of written } \\
\text { educational material }\end{array}$ & Usual care & $\begin{array}{l}\text { HbA1c, FBG, } \\
\text { BMI, SBP, DBP }\end{array}$ \\
\hline $\begin{array}{l}\text { Mehuys et al } \\
2011^{36} \\
\text { Belgium }\end{array}$ & $\begin{array}{l}\text { Cluster } \\
\text { randomized } \\
\text { controlled } \\
\text { study }\end{array}$ & $\begin{array}{l}\text { No. of Patients } \\
\text { (IG/CG):153/135 } \\
\text { Lost to follow-up } \\
\text { (IG/CG):5/3 } \\
\text { Age (IG/CG): } 63.0(40- \\
\text { 84)/62.3(45-79) } \\
\text { Gender (IG/CG) } \\
\text { female(\%):49/46.3 }\end{array}$ & 6 months & $\begin{array}{l}\text { Education on diabetes } \\
\text { and its complications, } \\
\text { medication and healthy } \\
\text { lifestyles, facilitation of } \\
\text { medication adherence, } \\
\text { and reminders about } \\
\text { annual eye and foot } \\
\text { examinations }\end{array}$ & $\begin{array}{l}\text { Usual } \\
\text { pharmacis } \\
\text { t care }\end{array}$ & $\begin{array}{l}\text { HbA1c, FBG, } \\
\text { medication } \\
\text { adherence, } \\
\text { diabetes } \\
\text { knowledge, } \\
\text { adherence to } \\
\text { self-care } \\
\text { activities }\end{array}$ \\
\hline $\begin{array}{l}\text { Mourao et al } \\
2013^{37} \\
\text { Brazil }\end{array}$ & $\begin{array}{l}\text { Parallel } \\
\text { randomized } \\
\text { controlled } \\
\text { study }\end{array}$ & $\begin{array}{l}\text { No. of patients } \\
\text { (IG/CG):65/64 } \\
\text { Lost to follow-up } \\
\text { (IG/CG):12/9 } \\
\text { Age (IG/CG): } \\
60.0[10.2] / 61.3 \text { [9.9] } \\
\text { Gender (IG/CG): } \\
68.0 \% / 66.0 \% \text { female }\end{array}$ & 6 months & $\begin{array}{l}\text { Pharmaceutical care, } \\
\text { providing patient } \\
\text { education about diabetes; } \\
\text { non pharmacological } \\
\text { issues, and } \\
\text { pharmacological } \\
\text { treatments proposals for } \\
\text { pharmacotherapy } \\
\text { changes forwarded to } \\
\text { PCP; }\end{array}$ & $\begin{array}{l}\text { Usual } \\
\text { health } \\
\text { care }\end{array}$ & $\begin{array}{l}\text { HbA1c, FBG, } \\
\text { SBP, DBP, TC, } \\
\text { LDL, HDL, TG, } \\
\text { BMI, medication } \\
\text { use, }\end{array}$ \\
\hline $\begin{array}{l}\text { Nascimento } \\
\text { et al } \\
201638 \\
\text { Portugal }\end{array}$ & $\begin{array}{l}\text { Parallel } \\
\text { randomized } \\
\text { controlled } \\
\text { study }\end{array}$ & $\begin{array}{l}\text { No. of Patients } \\
\text { (IG/CG):44/43 } \\
\text { Lost to follow-up } \\
\text { (IG/CG):0/0 } \\
\text { Age (IG/CG): } \\
74.2(5.4) / 72.3(4.5) \\
\text { Gender(IG/CG): } \\
43.2 / 41.9 \% \text { female }\end{array}$ & 6 months & $\begin{array}{l}\text { Individualized } \\
\text { pharmacotherapy } \\
\text { management service } \\
\text { including education about } \\
\text { diabetes and its } \\
\text { complication and } \\
\text { medication use, self- } \\
\text { management skills }\end{array}$ & NR & $\begin{array}{l}\text { HbA1c, FBG, } \\
\text { medication } \\
\text { adherence, } \\
\text { adherence to } \\
\text { self-care } \\
\text { activities }\end{array}$ \\
\hline
\end{tabular}




\begin{tabular}{|c|c|c|c|c|c|c|}
\hline $\begin{array}{l}\text { Samtia et al } \\
2013^{39} \\
\text { Pakistan }\end{array}$ & $\begin{array}{l}\text { Parallel } \\
\text { randomized } \\
\text { controlled } \\
\text { study }\end{array}$ & $\begin{array}{l}\text { No. of Patients (IG/CG): } \\
46.1(23-74) / 42.3(21-77) \\
\text { Lost to follow-up } \\
\text { (IG/CG):4/2 } \\
\text { Age (IG/CG): } \\
54(13) / 57(11) \\
\text { Gender (IG/CG): } \\
47.2 / 51.2 \% \text { female }\end{array}$ & 5 months & $\begin{array}{l}\text { Multifactorial } \\
\text { Intervention consisting of } \\
\text { education on diabetes, } \\
\text { medication use and } \\
\text { lifestyle modification, } \\
\text { counselling for } \\
\text { medication adherence } \\
\text { and SMBG education }\end{array}$ & NR & $\begin{array}{l}\text { HbA1c, FBG, } \\
\text { BMI, medication } \\
\text { adherence, } \\
\text { Diabetes } \\
\text { knowledge }\end{array}$ \\
\hline $\begin{array}{l}\text { Shao et al } \\
201740 \\
\text { China }\end{array}$ & $\begin{array}{l}\text { Parallel } \\
\text { randomized } \\
\text { controlled } \\
\text { study }\end{array}$ & $\begin{array}{l}\text { No. of Patients } \\
\text { (IG/CG):120/120 } \\
\text { Lost to follow-up } \\
\text { (IG/CG):20/21 } \\
\text { Age (IG/CG): } 58.86 \\
\pm 10.59 / 59.20 \pm 10.34 \\
\text { Gender (IG/CG): } \\
\text { 49/42.5\%female }\end{array}$ & 6 months & $\begin{array}{l}\text { Pharmaceutical care } \\
\text { including education on } \\
\text { diabetes and its } \\
\text { complications, medication } \\
\text { and healthy lifestyles, and } \\
\text { also on self-management } \\
\text { skills }\end{array}$ & Usual care & $\begin{array}{l}\text { HbA1c, FBS, } \\
\text { SBP, DBP, TC, } \\
\text { LDL-C, HDL-C, } \\
\text { TGs, BMI, } \\
\text { Medication } \\
\text { adherences, } \\
\text { self- care } \\
\text { activities }\end{array}$ \\
\hline $\begin{array}{l}\text { Siaw et al } \\
2017^{41} \\
\text { Singapore }\end{array}$ & $\begin{array}{l}\text { Parallel } \\
\text { randomized } \\
\text { controlled } \\
\text { study }\end{array}$ & $\begin{array}{l}\text { No. of Patients } \\
\text { (IG/CG):214/197 } \\
\text { Lost to follow-up } \\
\text { (IG/CG):0/0 } \\
\text { Age (IG/CG): } \\
59.2 \pm 8.2 / 60.1 \pm 8.1 \\
\text { Gender (IG/CG): } \\
47.7 / 39.1 \% \text { female }\end{array}$ & 6 months & $\begin{array}{l}\text { Multidisciplinary } \\
\text { collaborative care } \\
\text { including education on } \\
\text { diabetes and its } \\
\text { complications, and on } \\
\text { self-management skills }\end{array}$ & Usual care & $\begin{array}{l}\text { HbA1c, cost- } \\
\text { effective } \\
\text { analysis }\end{array}$ \\
\hline $\begin{array}{l}\text { Taveira et al } \\
2010^{42} \\
\text { USA }\end{array}$ & $\begin{array}{l}\text { Parallel } \\
\text { randomized } \\
\text { controlled } \\
\text { study }\end{array}$ & $\begin{array}{l}\text { No. of Patients } \\
\text { (IG/CG):64/54 } \\
\text { Lost to follow-up } \\
\text { (IG/CG):6/3 } \\
\text { Age (IG/CG):62.2 } \\
(10.3) / 66.8(10.2) \\
\text { Gender (IG/CG) } \\
\text { female(\%): } 8.6 / 0\end{array}$ & (a) & $\begin{array}{l}\text { Part of multidisciplinary } \\
\text { education intervention } \\
\text { regarding self-care } \\
\text { behaviours and } \\
\text { behavioural and } \\
\text { pharmacological } \\
\text { interventions, including } \\
\text { medication change }\end{array}$ & Usual care & $\begin{array}{l}\text { HbA1c, SBP, } \\
\text { DBP, LDL, non- } \\
\text { HDL, BMI, } \\
\text { tobacco use, } \\
\text { CHD risk factor, } \\
\text { adherence to } \\
\text { self-care } \\
\text { behaviours }\end{array}$ \\
\hline $\begin{array}{l}\text { Tourkmani } \\
\text { et al } \\
201643 \\
\text { Saudi Arabia }\end{array}$ & $\begin{array}{l}\text { Parallel } \\
\text { randomized } \\
\text { controlled } \\
\text { study }\end{array}$ & $\begin{array}{l}\text { No. of Patients } \\
\text { (IG/CG):140/122 } \\
\text { Lost to follow-up } \\
\text { (IG/CG):73/8 } \\
\text { Age (IG/CG): } 55.12 \\
\text { (12.76)/56.06 (11.08) } \\
\text { Gender (IG/CG):60/66.4 }\end{array}$ & 9 months & $\begin{array}{l}\text { Education program on } \\
\text { diabetes and its } \\
\text { complications, medication } \\
\text { and healthy lifestyles, and } \\
\text { also on self-management } \\
\text { skills, provision of written } \\
\text { educational material }\end{array}$ & $\begin{array}{l}\text { Standard } \\
\text { diabetic } \\
\text { care }\end{array}$ & $\begin{array}{l}\text { HbA1c, SBP, } \\
\text { DBP, LDL, HDL, } \\
\text { TC, TG BMI, }\end{array}$ \\
\hline $\begin{array}{l}\text { Wishah et al } \\
2015^{44} \\
\text { Jordan }\end{array}$ & $\begin{array}{l}\text { Parallel } \\
\text { randomized } \\
\text { controlled } \\
\text { study }\end{array}$ & $\begin{array}{l}\text { No. of patients } \\
\text { (IG/CG):52/54 } \\
\text { Lost to follow-up } \\
\text { (IG/CG):2/3 } \\
\text { Age (IG/CG): } \\
52.9[9.6] / 53.2[11.2] \\
\text { Gender (IG/CG): } \\
61.5 \% / 51.9 \% \text { female }\end{array}$ & 6 months & $\begin{array}{l}\text { Pharmaceutical care, } \\
\text { including optimization of } \\
\text { drug therapy; education } \\
\text { and counselling on } \\
\text { diabetes and medication; } \\
\text { enhancement of } \\
\text { adherence to medication } \\
\text { and self-care activities; } \\
\text { and provision of } \\
\text { educational leaflet and } \\
\text { brochures }\end{array}$ & $\begin{array}{l}\text { Usual care } \\
\text { provided } \\
\text { by the } \\
\text { medical } \\
\text { and } \\
\text { nursing } \\
\text { staff }\end{array}$ & $\begin{array}{l}\text { HbA1c, FBG, TC, } \\
\text { LDL, HDL, TG, } \\
\text { BMI, diabetes } \\
\text { knowledge, } \\
\text { medication } \\
\text { adherence, } \\
\text { diabetes self- } \\
\text { care activities }\end{array}$ \\
\hline
\end{tabular}

IG - intervention group; CG -control group; HRQoL- health related quality of life; BG-blood glucose; SBP - systolic blood pressure; DBP- diastolic blood pressure; TC - total cholesterol; LDL - low-density lipoprotein cholesterol; HDL - high- density lipoprotein cholesterol; TG triglycerides; BMI - body mass index; DQoL - diabetic quality of life; SIMS - satisfaction of information received about medicines; FBG - fasting blood glucose 
Various guidelines for diabetes recommend that a target HbA1c of a diabetic patient should be $7 \%$ or less. In order to decrease the chances of diabetic complication in those patients who have HbA1c more than $7 \%$, it is recommended to reduce the HbA1c up to $1 \%$ or more. Thus, a tight control of FBS and random blood sugar (RBS) is needed to decrease diabetes complications. ${ }^{17}$ Thus, pharmacist intervention is needed to reduce the FBS and RBS of patients as shown in the included studies of analysis. HbA1c was considered as an outcome measure in 24 studies. A1c mean value decreased in the intervention group during the follow-up period in all studies. Further analysis revealed that larger effect was made by studies which involved pharmacist-based diabetes education, followed by studies which involved pharmacistbased diabetes education plus pharmaceutical care and studies in which diabetes education was provided by health care team involving pharmacist. 46

Regarding the blood pressure, in this review it reveals that in comparison with the control, 11 out of 13 studies demonstrated a significant reduction in the blood pressure in the intervention group 21,25,28,30-33,35,37,40,42,43,44. The American Association of Clinical Endocrinologists and American College of Endocrinology CPGs on diabetes recommends that the target BP of diabetics should be less than 140/80-90 mm $\mathrm{Hg}$ to decrease the risk of microvascular and macrovascular complications. ${ }^{23}$ According to Indian guidelines, currently recommend the standard BP target of $<140 \mathrm{~mm}$ systolic and $<90 \mathrm{~mm}$ in patients with diabetes to decrease the chances of complications.

Ten studies described total cholesterol as an outcome measure $21,22,24,28,31,32,37.40,43,44$. In all of these studies, there was a reduction in the intervention group from baseline to final follow up, and seven studies reported a greater improvement in this outcome in comparison with the control group. Regarding LDL cholesterol, 14 studies reported data on this outcome, and all of them demonstrated a decrease in the intervention group from baseline to final follow-up $20-22,24-$ $26,28,31,32,37.40,42-44$. Ten studies reported a greater reduction in this outcome in the intervention group compared with the control group. Among the 11 studies that reported HDL cholesterol as an outcome measure, 10 studies described an increase in the intervention group from baseline to final follow-up $21,22,24,31,32,37,40,42,43,44$ and one study observed a decrease in the parameter ${ }^{28}$. Guidelines of various countries such as Australia, UK, USA, and Malaysia strongly recommend the use of a lipid-profile control medication such as a statin if it is not contraindicated to decrease the risk of cardiovascular disease (CVD) development ${ }^{17}$. Although the chances of development of diabetes with statin use are reported,47,48 many meta-analyses on randomized trials reported the advantages of statins to decrease the $\mathrm{CV}$ risks. ${ }^{17,49}$

Fifteen studies described BMI as an outcome measure, in all studies mean BMI decreased in the intervention group from baseline to final follow-up.21-24,28,30-32,35, 37, 39,40, 42-44

CHD risk was predicted among five of the included studies in diabetic patients $20,24,31,39,42$. The method used to estimate this risk varied between studies. All 5 studies recorded a decrease in CHD risk in the intervention group from baseline to final follow-up and reported a greater improvement in this group compared with the control group. Two studies used the United Kingdom Prospective Diabetes Study (UKPDS) risk engine, 31, 42 Two studies used the Framingham prediction method 20,39 , and one study used an equation specifically validated for the Hong Kong population ${ }^{24}$. All the models incorporated the variables age, sex, and smoking status ${ }^{6}$.the equation validated for the Hong Kong population also includes the duration of diabetes 24 . while the Framingham prediction model considers the presence or absence of diabetes as a variable. 50 In addition to the duration of diabetes, the UKPDS risk engine also incorporates the A1c mean values. Regarding blood pressure, the UKPDS risk engine and the British National Formulary prediction charts include systolic blood pressure, while the Framingham prediction model integrates systolic and diastolic blood pressure. As for the lipid profile, the UKPDS risk engine and the British National Formulary prediction charts consider the total cholesterol/HDL cholesterol ratio as a variable, while the Framingham prediction model only includes HDL cholesterol, and the equation validated for the Hong Kong population incorporates non-HDL cholesterol. In addition to the previously mentioned variables, the UKPDS risk engine also considers ethnicity as a variable, and the equation validated for the Hong Kong population integrates glomerular filtration rate and urinary albumin to creatinine ratio. ${ }^{6}$

Adherence to medications was measured in 12 studies $22-$ $24,28,30,31,33,36,38,39,40,44$. The methods used to measure this outcome varied between the studies. Self-reported adherence was used to measure this outcome almost in all studies, while one study used pill count or prescription refill rate in combination with self-reported adherence ${ }^{36}$. Eleven studies revealed an improvement in medication adherence in the intervention group from baseline to final follow-up.

Health-Related Quality of life was measured in six studies.2023.25,33 Five studies used the validated EuroQol-5 Dimension questionnaire which can be applied in different health conditions and diseases and one study used generic and diabetes-specific tools ${ }^{21}$ to measure this outcome. All studies reported an improvement in HRQoL in the intervention group from baseline to final follow-up, which was greater than that observed in the control group.

The evidence regarding the cost-effectiveness of pharmacist interventions was limited. In fact, only 3 studies conducted an economic analysis. Chan et al. (2012) estimated the costeffectiveness of the pharmacist care program being studied based on CHD risk reduction and the direct cost of time spent by the pharmacist in counselling and associated administrative work. ${ }^{24}$ The estimated potential saving in costs was $\$ 5,086.30$ USD per patient. ${ }^{24}$ Adibe et al. (2013) conducted a cost-utility analysis of the pharmaceutical care intervention implemented. ${ }^{20}$ The total cost per patient per year was $\$ 326.00$ USD for the control group and $\$ 394.00$ USD for the intervention group. ${ }^{20}$ Siaw et al. (2017) also conducted a cost-effectiveness analysis for the pharmacist intervention being studied. ${ }^{41}$ The 6 -month mean cost for direct outpatient diabetes- related care was US $\$ 516.77$ for the intervention arm and US\$607.78 for the control arm ( $\mathrm{p}<$ .001). Compared to the control arm, an average cost savings of US\$91.01 per patient over 6 months was achieved by the intervention arm. ${ }^{41}$ This is in accordance with the findings from the other 2 systematic reviews that evaluated the effects of pharmacist interventions on patients with diabetes, in which a small proportion of studies assessed the costeffectiveness of the interventions under study. ${ }^{6}$ However, given the current resource restraints in the health care systems, and in order to inform policymakers and influence their decisions towards widespread implementation of pharmacist interventions on the management of type 2 diabetes, cost-utility studies proving the cost-effectiveness of such interventions are of the utmost importance. Indeed, a comprehensive evaluation of pharmaceutical services has to consider clinical and humanistic outcomes, as well as economic outcomes. ${ }^{51}$ 


\section{CONCLUSION}

This review demonstrates a comprehensive evidence on the pharmacist-led interventions is associated with better glycaemic control and medication adherence. This narrative review supports a potential role of the pharmacists in diabetic care to help and support other healthcare professionals to achieve the target therapy outcomes. Therefore, they are very valuable to the care of such patients and should be considered and involved in diabetes care to help patients more effectively control their disease.

Overall, our review revealed that the pharmacist-led group intervention program was an efficacious and sustainable collaborative care approach to manage diabetes, selfmanagement interventions and reduce associated cardiovascular risk.

ACKNOWLEDGEMENT: The authors wishes to acknowledge Vinayaka Missions College of Pharmacy, Salem, Tamil Nadu-636008 and Ahalia School of Pharmacy, Palakkad, Kerela-678557, for providing their library support and other logistic facilities in framing the review article.

CONFLICTS OF INTEREST: The author declares no conflict of interest.

\section{REFERENCES}

1. International Diabetes Federation. IDF Diabetes Atlas. 9th ed. Brussels, Belgium: International Diabetes Federation. 2019. Available at: http://www. idf.org/diabetesatlas. Accessed May 14, 2020.

2. Benedict AW, Spence MM, Sie JL et al. Evaluation of a Pharmacist-Managed diabetes program in a primary care setting within an integrated health care system. J Manag Care Spec Pharm.2018; 24(2):114-22.

3. Wu Y, Ding Y, Tanaka Y, Zhang W et al. Risk factors contributing to type 2 diabetes and recent advances in the treatment and prevention. Int J Med Sci. 2014; 11(11):1185-200.

4. Siaw M Y L, Malone D C, Ko Y et al. Cost-effectiveness of multidisciplinary collaborative care versus usual care in the management of high-risk patients with diabetes in Singapore: Short term results from a randomized controlled trial. J Clin Pharm Ther. 2018;1-9.

5. Marx MC, Dahal A, Jennings B et al. The effect of a diabetes collaborative care management program on clinical and Economic outcomes in patients with type 2 diabetes. J Manag Care Spec Pharm. 2015; 21(6):452-68.

6. Pousinho S, Margado M, Falcao A, Aves G. Pharmacist intervention in the management of type 2 diabetes mellitus: A systematic review of randomised controlled trials. J Manag Care Spec Pharm.2016; 22(5):493-515.

7. Eliasson B, Cederholm J, Nilsson P, Gudbjornsdottir S; Steering Committee of the Swedish National Diabetes Register. The gap between guidelines and reality: type 2 diabetes in a National Diabetes Register 1996-2003. Diabet Med. 2005; 22(10):142026.

8. Antoine SL, Pieper D, Mathes T et al. Improving the adherence of type 2 diabetes mellitus patients with pharmacy care: a systematic review of randomized controlled trials. BMC. 2014; 14:53.

9. Wubben DP, Vivian EM. Effects of pharmacist outpatients' interventions on adults with diabetes mellitus: a systematic review. Pharmacotherapy.2008; 28:421-436.

10. Tapp H, Phillips SE, Waxman D, et al. Multidisciplinary team approach to improved chronic care management for diabetic patients in an urban safety net ambulatory care clinic. J Am Board Fam Med.2012; 25:245-246.

11. American Diabetes Association. Standards of medical care in diabetes -2017.Diabetes Care. 2017; 40 (suppl 1): S1-S32.

12. Presley B, Groot W, Pavlova M. Pharmacy-led interventions to improve medication adherence among adults with diabetes: A Systematic review and meta- analysis. Research in Social and Administrative Pharmacy. 2019; 15:1057-1067.
13. McClean MT, McElnay JC, Andrews J. The importance of patients education and patient involvement in the treatment of diabetes. Pharm J. 2000; 265:108-10.

14. Grossman S. Management of type 2 diabetes mellitus in the elderly: role of the pharmacist in a multidisciplinary health care team. J Multidiscip Healthc.2011; 4:149-154.

15. van Eikenhorst L, Taxis K, van Dijk L, de Gier H. Pharmacist led self-management interventions to improve diabetes outcomes: a systematic literature review and meta-analysis. Front Pharmacol 2017; 8:891.

16. Hassali MA, Nazir SU, Saleem F, Masood I. Literature review: pharmacists' interventions to improve control and management in type 2 diabetes mellitus. Altern Ther Health Med 2015; 21:2835.

17. Iqbal MZ, Khan AH, Iqbal MS, Sulaiman SAS. A review of pharmacist-led interventions on diabetes outcomes: An observational analysis to explore diabetes care opportunities for pharmacists. J Pharm Bioall Sci. 2019; 11:299-309.

18. Rajpathak SN, Kumbhani DJ, Crandall J, Barzilai N, Alderman M, Ridker PM. Statin therapy and risk of developing type 2 diabetes: a meta-analysis. Diabetes Care 2009; 32:1924-9.

19. DeCoster VA. Challenges of type 2 diabetes sand role of health care social work: a neglected area of practice. Health Soc Work 2001; 26:26-37.

20. Adibe MO, Ukwe CV, Aguwa CN. The impact of pharmaceutical care intervention on the quality of life of Nigerian patients receiving treatment for type 2 diabetes. Value Health Reg Issues. 2013; 2(2):240-47.

21. Ali M, Schifano F, Robinson $P$, et al. Impact of community pharmacy diabetes monitoring and education programme on diabetes management: a randomized controlled study. Diabet Med. 2012; 29(9):326-33.

22. Butt M., Mhd Ali A, Bakry MM. and Mustafa N. Impact of a pharmacist-led diabetes mellitus intervention on HbA1c, medication adherence and quality of life: a randomised controlled study. Saudi Pharm. J. 2016; 24: 40-48.

23. Cani CG, Lopes LS, Queiroz M, and Nery M. Improvement in medication adherence and self-management of diabetes with a clinical pharmacy program: a randomized controlled trial in patients with type 2 diabetes undergoing insulin therapy at a teaching hospital. Clinics. 2015; 70: 102-106.

24. Chan CW, Siu SC, Wong CK, Lee VW. A pharmacist care program: positive impact on cardiac risk in patients with type 2 diabetes. J Cardiovasc Pharmacol Ther. 2012; 17(1):57-64.

25. Cohen LB, Taveira TH, Khatana SA, Dooley AG, Pirraglia PA, Wu WC. Pharmacist-led shared medical appointments for multiple cardiovascular risk reduction in patients with type 2 diabetes. Diabetes Educ. 2011; 37(6): 801-12.

26. Doucette WR, Witry MJ, Farris KB, and McDonough RP. Community pharmacist-provided extended diabetes care. Ann. Pharm.2009; 43: 882-889.

27. Farsaei S, Sabzghabaee AM, Zargarzadeh AH, Amini M. Effect of pharmacist-led patient education on glycemic control of type 2 diabetics: a randomized controlled trial. J Res Med Sci. 2011; 16(1):43-49.

28. Jarab AS, Alqudah SG, Mukattash TL, Shattat G, Al-Qirim T. Randomized controlled trial of clinical pharmacy management of patients with type 2 diabetes in an outpatient diabetes clinic in Jordan. J Manag Care Pharm. 2012; 18(7):516-26.

29. Jameson JP, and Baty PJ. Pharmacist collaborative management of poorly controlled diabetes mellitus: a randomized controlled trial. Am. J. Manag. Care.2010; 16: 250-255.

30. Jahangard-Rafsanjani Z, Sarayani A, Nosrati M, Saadat N, Rashidian, Hadjibabaie,M, et al. Effect of a community pharmacist delivered diabetes support program for patients receiving specialty medical care: a randomized controlled trial. Diabet. Educ. 2015; 41: 127-135.

31. Korcegez EI, Sancar M, and Demirkan K. Effect of a pharmacist led program on improving outcomes in patients with type 2 diabetes mellitus from Northern Cyprus: a randomized controlled trial. J. Manage. Care Special. Pharm. 2017; 23:573582.

32. Kraemer DF, Kradjan WA, Bianco TM, Low JA. A randomized study to assess the impact of pharmacist counselling of employer-based health plan beneficiaries with diabetes: the EMPOWER study. J Pharm Pract. 2012; 25:169-79.

33. Kjeldsen LJ, Bjerrum L, Dam P, Larsen BO, Rossing C, Søndergaard B. Safe and effective use of medicines for patients 
with type 2 diabetes: a randomized controlled trial of two interventions delivered by local pharmacies. Res Social Adm Pharm. 2015; 11(1):47-62.

34. Lau BT, Ismail SZ, Ng SY, Mohmmad N. Impact of pharmacist led diabetes program on glycated haemoglobin and diabetes related hospitalizations in a district-level hospital: a pilot retrospective cohort study. Int J Adv Life Sci Res 2018; 1:26-36.

35. Lim PC, Lim K, Embee ZC, Hassali MA, Thiagarajan A, Khan TM. Study investigating the impact of pharmacist involvement on the outcomes of diabetes medication therapy adherence program Malaysia. Pak J Pharm Sci. 2016; 29:595-601.

36. Mehuys E, Van Bortel L, De Bolle L, Van Tongelen I, Annemans L, Remon JP, et al. Effectiveness of a community pharmacist intervention in diabetes care: a randomized controlled trial. J Clin Pharm Ther. 2011; 36:602-13.

37. Mourão AO, Ferreira WR, Martins MA, Reis AM, Carrillo MR, Guimarães AG, et al. Pharmaceutical care program for type 2 diabetes patients in Brazil: a randomised controlled trial. Int J Clin Pharm. 2013; 35:79-86.

38. Nascimentoa T, Braz N, Gomes E, Fernandez-Arche A De La Puerta R. Self-care improvement after a pharmaceutical intervention in elderly type 2 diabetic patients. Curr Diabetes Rev. 2015; 12:120-8.

39. Samtia AM, Rasool MF, Ranjha NM, Usman F, Javed I. A multifactorial intervention to enhance adherence to medications and disease-related knowledge in type 2 diabetic patients in Southern Punjab, Pakistan. Trop J Pharm Res. 2013; 12:851-6.

40. Shao H, Chen G, Zhu C, Chen Y, Liu Y, He Y, et al. Effect of pharmaceutical care on clinical outcomes of outpatients with type 2 diabetes mellitus. Patient Prefer Adherence. 2017; 11:897-903.

41. Siaw MYL, Ko Y, Malone DC, Tsou KYK, Lew J, Foo D, et al. Impact of pharmacist-involved collaborative care on the clinical, humanistic and cost outcomes of high-risk patients with type 2 diabetes (IMPACT): a randomized controlled trial. J. Clin. Pharm. Ther. 2017; 42, 475-482.
42. Taveira TH, Friedmann PD, Cohen LB, Dooley AG, Khatana SA, Pirraglia PA, et al. Pharmacist-led group medical appointment model in type 2 diabetes. Diabetes Educ. 2010; 36:109-17.

43. Tourkmani AM, Hassali MA, Alharbi TJ, Alkhashan HI, Alobikan $\mathrm{AH}$, Bakhiet $\mathrm{AH}$, et al. Impact of Ramadan focused education program on hypoglycemic risk and metabolic control for patients with type 2 diabetes. Patient Prefer. Adherence. 2016; 10:1709-1717.

44. Wishah RA, Al-Khawaldeh OA, Albsoul AM. Impact of pharmaceutical care interventions on glycemic control and other health-related clinical outcomes in patients with type 2 diabetes: randomized controlled trial. Diabetes Metab Syndr. 2015;9(4):271-76.

45. Liang X, Wang Q, Yang X, Cao J, Chen J, Mo X, et al. Effect of mobile phone intervention for diabetes on glycaemic control: A meta-analysis. Diabet Med. 2011; 28:455-63.

46. Bukhsh A, Khan $\mathrm{T}$ M, Lee $\mathrm{S}$ W H, Lee L H, et al. Efficacy of Pharmacist-based Diabetes Educational interventions on clinical outcomes of adults with type 2 diabetes mellitus: A Network Meta-Analysis. Front. Pharmacol. 2018; 9:339.

47. Liew SM, Lee PY, Hanafi NS, Ng CJ, Wong SS, Chia YC, et al. Statins use is associated with poorer glycaemic control in a cohort of hypertensive patients with diabetes and without diabetes. Diabetol Metab Syndr 2014; 6:53.

48. Sattar N, Preiss D, Murray HM, Welsh P, Buckley BM, de Craen AJ, et al. Statins and risk of incident diabetes: a collaborative metaanalysis of randomised statin trials. Lancet 2010; 375:735-42.

49. Pradhan A, Libby P. Cardiovascular benefits and diabetes risks of statin therapy in primary prevention. Lancet $2012 ; 380: 565-71$.

50. Wilson PW, D'Agostino RB, Levy D, Belanger AM, Silbershatz $\mathrm{H}_{\text {, }}$ Kannel WB. Prediction of coronary heart disease using risk factor categories. Circulation. 1998; 97(18):1837-47.

51. Schulz KF, Altman DG, Moher D; CONSORT Group. CONSORT 2010 statement: updated guidelines for reporting parallel group randomised trials. BMJ. 2010; 340:c332. 